PROCEEDINGS OF THE

AMERICAN MATHEMATICAL SOCIETY

Volume 127, Number 12, Pages 3633-3642

S 0002-9939(99)05204-1

Article electronically published on May 17, 1999

\title{
GLOBAL ATTRACTOR IN AUTONOMOUS COMPETITIVE LOTKA-VOLTERRA SYSTEMS
}

\author{
ZHANYUAN HOU
}

(Communicated by Michael Handel)

\begin{abstract}
For autonomous Lotka-Volterra systems modelling the dynamics of $N$ competing species, a new condition has been found to prevent a particular species from dying out. Based on this condition, criteria have been established for all or some of the $N$ species to stabilise at a steady state whilst the others, if any, die out.
\end{abstract}

\section{INTRODUCTION}

Consider the autonomous Lotka-Volterra system

$$
\dot{x}_{i}=b_{i} x_{i}\left(1-\alpha_{i} x\right) \quad\left(i \in I_{N}\right),
$$

where $\dot{x}_{i}=d x_{i} / d t, b_{i}>0, I_{m}=\{1,2, \ldots, m\}$ for any integer $m \geq 1, N \geq 3$, $x=\left(x_{1}, x_{2}, \ldots, x_{N}\right)^{T} \in \mathbb{R}^{N}$ and $\alpha_{i}=\left(a_{i 1}, a_{i 2}, \ldots, a_{i N}\right)$ with $a_{i i}>0$ and $a_{i j} \geq 0$ $(i \neq j)$. Since $(1.1)$ is well known as a model of a community of $N$ mutually competing species, $x_{i}$ denoting the population size of the $i$ th species at time $t$, we adopt the usual restriction of $x$ to the positive cone $\mathbb{R}_{+}^{N}$.

We are interested in the existence of a global attractor $x^{*} \in \mathbb{R}_{+}^{N}$. If $x^{*} \in \partial \mathbb{R}_{+}^{N}$ (i.e., the boundary of $\mathbb{R}_{+}^{N}$ ), then some of the $N$ species will eventually die out whilst the others will coexist and stabilise at a steady state. If $x^{*} \in \operatorname{int} \mathbb{R}_{+}^{N}$ (i.e., $\mathbb{R}_{+}^{N} \backslash \partial \mathbb{R}_{+}^{N}$ ), however, no extinction will occur and all of the species will stabilise at $x^{*}$. A simple and frequently used condition for (1.1) to have a global attractor $x^{*} \in \operatorname{int} \mathbb{R}_{+}^{N}$ is

$$
\frac{a_{i 1}}{a_{11}}+\frac{a_{i 2}}{a_{22}}+\cdots+\frac{a_{i N}}{a_{N N}}<2
$$

for all $i \in I_{N}$ (see Kaykobad [6], Gopalsamy and Ahlip [5] and Gopalsamy [4, pp. 294-297]). For the existence of a global attractor $x^{*} \in \partial \mathbb{R}_{+}^{N}$, Zeeman [9] gives a condition for the survival of only one species and Ahmad and Lazer [2] obtain a criterion for the extinction of only one species. Bridging the gap between the above extreme cases, Montes de Oca and Zeeman [7] prove that the species $x_{1}, \ldots, x_{r}$ stabilise at $\left(x_{1}^{*}, \ldots, x_{r}^{*}\right)^{T} \in \operatorname{int} \mathbb{R}_{+}^{r}$ whereas $x_{r+1}, \ldots, x_{N}$ go extinct provided (1.2)

Received by the editors February 19, 1998.

1991 Mathematics Subject Classification. Primary 34D45; Secondary 34A26, 92D25.

Key words and phrases. Lotka-Volterra, global attractor, autonomous systems, competition.

(C)1999 American Mathematical Society 
holds for $i \in I_{r}$ and

$$
\forall k \in I_{N} \backslash I_{r}, \quad \exists i_{k}<k \quad \ni \quad \forall j \in I_{k}, \quad a_{i_{k} j}<a_{k j},
$$

where the symbol " $\ni$ " reads "such that".

We observe that (1.2) for some $i \in I_{N}$ is employed to prevent the species $x_{i}$ from extinction while (1.3) for $k=N$ is to drive $x_{N}$ to extinction (cf. Montes de Oca and Zeeman [8, Lemma 3.1]). We also notice that these ideas, especially (1.2), are extended to nonautonomous (see [7, 8], Ahmad [1], Ahmad and Lazer [3]) and retarded autonomous $([5]$ and $[4, \S 4.1-\S 4.3])$ systems. No doubt the popularity of (1.2) is attributed to its conciseness. Nonetheless, as a means of preserving and stabilising $x_{i}$ at some $x_{i}^{*}>0,(1.2)$ is too restrictive and is far from necessary. When $N=3$, van den Driessche and Zeeman [10] provide a simpler criterion for the global attractivity of a steady state $x^{*} \in \operatorname{int} \mathbb{R}_{+}^{N}$ assuming the existence of $x^{*}$. For the general system (1.1), however, it seems that (1.2) for all $i \in I_{N}$ is the only condition available for both the existence of a steady state $x^{*} \in \operatorname{int} \mathbb{R}_{+}^{N}$ and its global attractivity.

The purpose of this paper is to find new conditions that are less restrictive than (1.2) and (1.3) for the existence of a global attractor $x^{*} \in \mathbb{R}_{+}^{N}$.

\section{Main Results}

For any $\alpha_{0}=\left(a_{01}, a_{02}, \ldots, a_{0 N}\right) \neq 0$ with all $a_{0 j} \geq 0$, the set

$$
\gamma_{0}=\left\{x \in \mathbb{R}_{+}^{N}: \alpha_{0} x=1\right\}
$$

can be viewed as an $(N-1)$-dimensional plane in $\mathbb{R}_{+}^{N}$. Let $\gamma_{i}$ be given by $(2.1)$ with the replacement of 0 by $i \in I_{N}$ and let

$$
Y=\left(\frac{1}{a_{11}}, \frac{1}{a_{22}}, \ldots, \frac{1}{a_{N N}}\right)^{T} .
$$

From system (1.1) itself we perceive that the $\gamma_{i}$, together with $Y$, will play an important role in determining the dynamics of (1.1). Define $y^{S}$ for $y \in \mathbb{R}_{+}^{N}$ and $S \subseteq I_{N}$ by $y_{i}^{S}=y_{i}$ if $i \in S$ and $y_{i}^{S}=0$ otherwise. Then, for any nonempty $S \subseteq I_{N}$ and every $j \in S, \alpha_{j} Y^{S} \geq 1$. In particular, $\alpha_{i} Y \geq 1$ for all $i \in I_{N}$.

Condition 2.1. For a fixed $i \in I_{N}$ and every pair of $m \in I_{N} \backslash\{i\}$ and $S \subseteq$ $I_{N} \backslash\{i, m\}$ such that

$$
\alpha_{i} Y^{S}<1 \leq \alpha_{i} Y^{S \cup\{m\}},
$$

every $\alpha_{j}(j \neq i)$ in (1.1) satisfies

$$
\text { either } \quad 1 \leq \alpha_{j} Y^{S} \quad \text { or } \quad \frac{1}{a_{j m}}\left(1-\alpha_{j} Y^{S}\right)<\frac{1}{a_{i m}}\left(1-\alpha_{i} Y^{S}\right) \text {. }
$$

Remark 2.2. Condition 2.1 for some $i \in I_{N}$ implies that

$$
a_{i j}<a_{j j} \quad\left(j \in I_{N} \backslash\{i\}\right) .
$$

In fact, if $a_{i m} \geq a_{m m}$ for some $m \neq i$, i.e., $(2.3)$ holds for this $m$ and $S=\emptyset$, then (2.4) leads to a contradiction $a_{i m}<a_{m m}$. This also shows that $S \neq \emptyset$ for any pair $(m, S)$ satisfying $(2.3)$ and $(2.4)$. 

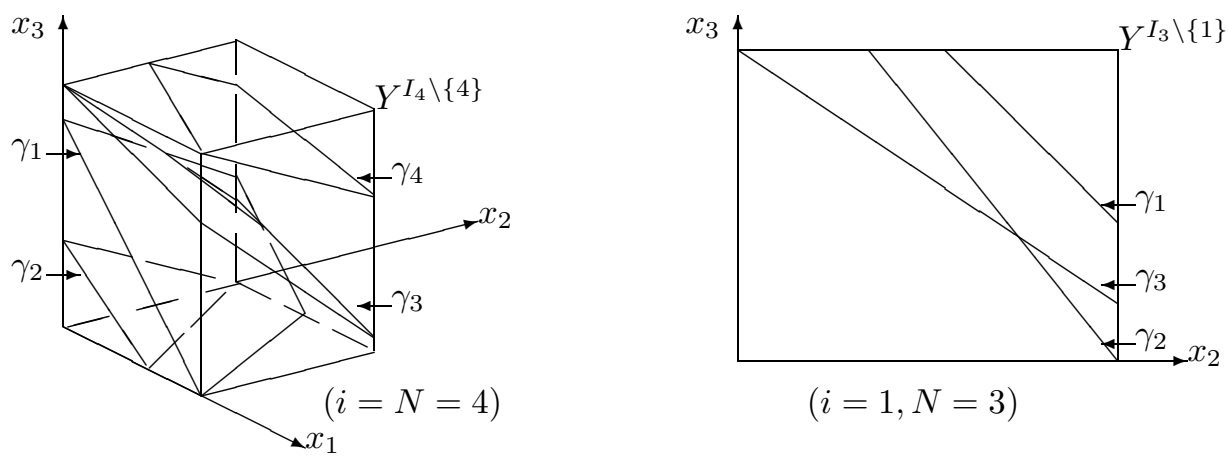

Figure 1. The left (right) satisfies Condition $2.1((2.8)$ for $j \in$ $\left.I_{3} \backslash\{1\}\right)$ but does not satisfy (2.8) for $j \in I_{2}((1.2))$.

The following are preliminaries towards a geometric interpretation of Condition 2.1. The set $\gamma_{0}$ given by (2.1) is convex in the sense that $c_{1} x^{1}+c_{2} x^{2} \in \gamma_{0}$ whenever $x^{i} \in \gamma_{0}$ and $c_{i} \geq 0\left(i \in I_{2}\right)$ with $c_{1}+c_{2}=1$. We say that $x \in \mathbb{R}_{+}^{N}$ is below (on or above) $\gamma_{0}$ if $\alpha_{0} x<1\left(=1\right.$ or $>1$ ). A set $D \subset \mathbb{R}_{+}^{N}$ is said to be below (on or above) $\gamma_{0}$ if every point in $D$ is so. For any $y^{1}, y^{2} \in \mathbb{R}_{+}^{N}$ with $y^{1} \leq y^{2}$ (i.e., $y_{j}^{1} \leq y_{j}^{2}$ for all $\left.j \in I_{N}\right)$, the cell

$$
\left[y^{1}, y^{2}\right]=\left\{x \in \mathbb{R}_{+}^{N}: y^{1} \leq x \leq y^{2}\right\}
$$

is $k$-dimensional if $y_{j}^{1}<y_{j}^{2}$ holds exactly for $k$ indices. Clearly, $\left[y^{1}, y^{2}\right]$ is a convex set too. Suppose that $y^{1}$ is below $\gamma_{0}$ whereas $y^{2}$ is on or above $\gamma_{0}$. Then there are pairs of $m \in I_{N}$ and $S \subseteq I_{N} \backslash\{m\}$ such that $y^{1}+\left(y^{2}-y^{1}\right)^{S}$ is below $\gamma_{0}$ whilst $y^{1}+\left(y^{2}-y^{1}\right)^{S \cup\{m\}}$ is on or above $\gamma_{0}$. Hence, for each such pair $(m, S)$, there is a $y(m, S) \in\left[y^{1}, y^{2}\right] \cap \gamma_{0}$ such that

$$
\left[y^{1}+\left(y^{2}-y^{1}\right)^{S}, y^{1}+\left(y^{2}-y^{1}\right)^{S \cup\{m\}}\right] \cap \gamma_{0}=\{y(m, S)\} .
$$

Let $P$ be the set of all such $y(m, S)$, so $P$ consists of the points of intersection of $\gamma_{0}$ and the edges of the cell $\left[y^{1}, y^{2}\right]$. Then, since $\left[y^{1}, y^{2}\right] \cap \gamma_{0}$ is convex, it can be shown that

$$
\left[y^{1}, y^{2}\right] \cap \gamma_{0}=\left\{\sum_{k} c_{k} x^{k}: c_{k} \geq 0, x^{k} \in P, \sum_{k} c_{k}=1\right\} .
$$

So $\left[y^{1}, y^{2}\right] \cap \gamma_{0}$ is the convex hull of $P$.

Remark 2.3. Condition 2.1 is equivalent to the following statement: Every set $\left[Y^{\emptyset}, Y^{I_{N} \backslash\{i\}}\right] \cap \gamma_{j}(j \neq i)$, the restriction of $\gamma_{j}$ to $\left[Y^{\emptyset}, Y^{I_{N} \backslash\{i\}}\right]$, is below the plane $\gamma_{i}$. In other words, $\left[Y^{\emptyset}, Y^{I_{N} \backslash\{i\}}\right] \cap \gamma_{i}$, if not empty, is above every plane $\gamma_{j}(j \neq i)$ (see Figure 1). This is obvious if $\alpha_{i} Y^{I_{N} \backslash\{i\}}<1$ as, in this case, $(m, S)$ satisfying (2.3) does not exist and the whole cell $\left[Y^{\emptyset}, Y^{I_{N} \backslash\{i\}}\right]$ is below $\gamma_{i}$. If $\alpha_{i} Y^{I_{N} \backslash\{i\}} \geq 1$, since $\alpha_{i} Y^{\emptyset}=0<1$, then (2.3) implies (2.6) with the replacements of $\gamma_{0}$ by $\gamma_{i}$ and $\left[y^{1}, y^{2}\right]$ by $\left[Y^{\emptyset}, Y^{I_{N} \backslash\{i\}}\right]$. For each pair $(m, S)$ satisfying (2.3) and every $j \in I_{N} \backslash\{i\},(2.4)$ holds if and only if $y(m, S)$ in (2.6) is above $\gamma_{j}$. From (2.7) with the appropriate replacement we see that Condition 2.1 holds if and only if $\left[Y^{\emptyset}, Y^{I_{N} \backslash\{i\}}\right] \cap \gamma_{i}$ is above $\gamma_{j}$ for all $j \in I_{N} \backslash\{i\}$.

We are now able to give an alternative of Condition 2.1 . 
Lemma 2.4. (i) Let $J \subseteq I_{N}$ with cardinality $|J|>1$. If Condition 2.1 is met for all $i \in J$, then

$$
\max \left\{0, \frac{a_{i j}}{a_{j j}}\left(1-\alpha_{j} Y^{I_{N} \backslash\{i, j\}}\right)\right\}<1-\alpha_{i} Y^{I_{N} \backslash\{i, j\}}
$$

for all $i, j \in J$ with $i \neq j$. (ii) Conversely, for a fixed $i \in I_{N}$, (2.8) for all $j \in I_{N} \backslash\{i\}$ implies Condition 2.1. (iii) Hence, Condition 2.1 is fulfilled for all $i \in I_{N}$ if and only if (2.8) holds for all $i, j \in I_{N}$ with $i \neq j$.

Proof. (i) Under Condition 2.1 for all $i \in J$, we suppose $\alpha_{i} Y^{I_{N} \backslash\{i, j\}} \geq 1$ for some $i, j \in J$ with $i \neq j$. Then $\left[Y^{\emptyset}, Y^{I_{N} \backslash\{i\}}\right] \cap \gamma_{i} \supseteq\left[Y^{\emptyset}, Y^{I_{N} \backslash\{i, j\}}\right] \cap \gamma_{i} \neq \emptyset$. By Remark 2.3, $\left[Y^{\emptyset}, Y^{I_{N} \backslash\{i, j\}}\right] \cap \gamma_{i}$ is above $\gamma_{j}$. On the other hand, as Condition 2.1 holds for $j$ and $\left[Y^{\emptyset}, Y^{I_{N} \backslash\{i, j\}}\right] \subset\left[Y^{\emptyset}, Y^{I_{N} \backslash\{j\}}\right]$, by Remark 2.3 again $\left[Y^{\emptyset}, Y^{I_{N} \backslash\{i, j\}}\right] \cap \gamma_{i}$ is below $\gamma_{j}$. This contradiction shows that $\alpha_{j} Y^{I_{N} \backslash\{i, j\}}<1$ for all $i, j \in J$ with $i \neq j$. Since $\alpha_{j} Y^{I_{N} \backslash\{i\}} \geq 1$, we have $\left[Y^{I_{N} \backslash\{i, j\}}, Y^{I_{N} \backslash\{i\}}\right] \cap \gamma_{j}=\{z\}$ where

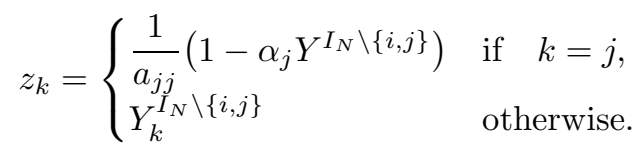

By Remark 2.3, $z$ is below $\gamma_{i}$, i.e.,

$$
\alpha_{i} z=\alpha_{i} Y^{I_{N} \backslash\{i, j\}}+a_{i j} z_{j}<1 .
$$

Then (2.8) follows for $i, j \in J$ with $i \neq j$.

(ii) If $\alpha_{i} Y^{I_{N} \backslash\{i\}}<1$, then $\left[Y^{\emptyset}, Y^{I_{N} \backslash\{i\}}\right]$ is below $\gamma_{i}$. By Remark 2.3, Condition 2.1 holds for this $i$. Suppose $\alpha_{i} Y^{I_{N} \backslash\{i\}} \geq 1$. Then, since (2.8) holds for $j \in I_{N} \backslash\{i\}$, for every pair $(m, S)$ satisfying (2.3) we must have $m \in I_{N} \backslash\{i\}$ and $S=I_{N} \backslash\{i, m\}$. For $j \in I_{N} \backslash\{i\}$, it is obvious that $\alpha_{j} Y^{S} \geq 1$ if $j \in S$. If $j \notin S$, then $j=m$ and

$$
\frac{1}{a_{m m}}\left(1-\alpha_{m} Y^{S}\right)<\frac{1}{a_{i m}}\left(1-\alpha_{i} Y^{S}\right)
$$

by (2.8). Therefore, (2.4) holds for $(m, S)$ satisfying (2.3) and $j \in I_{N} \backslash\{i\}$.

(iii) This is a combination of $(i)$ and $(i i)$.

Remark 2.5. If (1.2) holds for some $i \in I_{N}$, then

$$
0<1-\alpha_{i} Y^{I_{N} \backslash\{i\}}=1-\alpha_{i} Y^{I_{N} \backslash\{i, j\}}-\frac{a_{i j}}{a_{j j}} \quad(j \neq i)
$$

which, together with $-a_{j j}^{-1} a_{i j} \alpha_{j} Y^{I_{N} \backslash\{i, j\}} \leq 0$, implies (2.8) for all $j \in I_{N} \backslash\{i\}$. Hence, by Lemma 2.4 (ii), (1.2) also implies Condition 2.1. But the converses are not true (see Figure 1).

Theorem 2.6. Assume that (2.8) holds for all $i, j \in I_{N}$ with $i \neq j$. Then (1.1) has a global attractor $x^{*} \in \operatorname{int} \mathbb{R}_{+}^{N}$ with $x_{i}^{*} \in\left(0,1 / a_{i i}\right]$ for $i \in I_{N}$.

Example 2.7. For (1.1) with $\alpha_{1}=(1, \varepsilon, 2 \varepsilon), \alpha_{2}=(\varepsilon, 1, \varepsilon)$ and $\alpha_{3}=(2 \varepsilon, \varepsilon, 1)$, where $\varepsilon \in\left(\frac{1}{3}, \frac{3-\sqrt{5}}{2}\right)$, we have $\varepsilon^{2}-3 \varepsilon+1>0$ so that $(2.8)$ holds for all $i, j \in I_{3}$ with $i \neq j$. By Theorem 2.6, (1.1) has a global attractor $x^{*} \in \operatorname{int} \mathbb{R}_{+}^{3}$. It is obvious that (1.2) for $i=1$, i.e. $3 \varepsilon<1$, is not satisfied.

Remark 2.8. Remark 2.5 and Example 2.7 show that Theorem 2.6 suits a broader class of systems than the one using (1.2). As (2.8) is still concise enough, with little extra cost we have obtained a better result and thus achieved a goal set in $\S 1$. 
Theorem 2.9. Let $J \subset I_{N}$ with $1 \leq|J| \leq N-1$. Assume that Condition 2.1 holds for all $i \in J$ and

$$
\forall k \in I_{N} \backslash J, \quad \exists i_{k} \in J \quad \ni \quad \forall j \in J, \quad a_{i_{k} j} \leq a_{k j} .
$$

Then (1.1) has a global attractor $x^{*} \in \partial \mathbb{R}_{+}^{N}$ with $x_{\ell}^{*} \in\left(0,1 / a_{\ell \ell}\right]$ if $\ell \in J$ and $x_{\ell}^{*}=0$ otherwise.

Remark 2.10. We show that (1.3) implies (2.9) with $J=I_{r}$. For any $k \in I_{N} \backslash J$, let $i_{k}$ be given by (1.3). We need find an $\ell_{k} \leq r$ such that $a_{\ell_{k} j} \leq a_{k j}$ for all $j \in J$ so that (2.9) holds for this $k$. If $i_{k} \leq r$, then $\ell_{k}=i_{k}$ meets the requirement. If $r<i_{k}<k$, by (1.3) there is an $m_{k}<i_{k}$ such that $a_{m_{k} j}<a_{i_{k} j}<a_{k j}$ for all $j \in I_{i_{k}}$. We then take $\ell_{k}=m_{k}$ if $m_{k} \leq r$. If $m_{k}>r$, repeating the above process a number of times, we can always find the required $\ell_{k}$.

Replacing Condition 2.1 by (2.8) in Theorem 2.9, we have the following result.

Corollary 2.11. Let $J \subset I_{N}$ with $1 \leq|J| \leq N-1$. Assume that (2.8), for every $i \in J$ and all $j \in I_{N} \backslash\{i\}$, and (2.9) are satisfied. Then the conclusion of Theorem 2.9 holds.

Example 2.12. Consider (1.1) with $\alpha_{1}=\left(1, \frac{1}{2}, \frac{1}{3}, \frac{1}{3}\right), \alpha_{2}=\left(0,1, \frac{1}{3}, \frac{1}{3}\right), \alpha_{3}=$ $(1,1,1,0)$ and $\alpha_{4}=\left(2, \frac{1}{2}, \frac{1}{6}, 1\right)$. We can check that $(2.8)$, i.e.,

$$
\max \left\{0, a_{i j}\left(1-a_{j k}-a_{j \ell}\right)\right\}<1-a_{i k}-a_{i \ell} \quad\left(k, \ell \in I_{4} \backslash\{i, j\}, k \neq \ell\right),
$$

holds for $i \in I_{2}$ and $j \in I_{4} \backslash\{i\}$. Since $a_{21} \leq a_{31}, a_{22} \leq a_{32}, a_{11} \leq a_{41}$ and $a_{12} \leq a_{42}$, (2.9) with $J=I_{2}$ is satisfied. By Corollary 2.11, (1.1) has a global attractor $x^{*} \in \partial \mathbb{R}_{+}^{4}$ with $x_{3}^{*}=x_{4}^{*}=0$ and $x_{i}^{*} \in(0,1]$ for $i \in I_{2}$. Note that $a_{11}+a_{12}+a_{13}+a_{14}>2, a_{13}>a_{43}, a_{23}>a_{43}$ and $a_{33}>a_{43}$. So neither (1.2) for $i=1$ nor (1.3) for $k=4$ is met.

Remark 2.13. From Example 2.12 and Remarks 2.5 and 2.10 we see that Theorem 2.9 and Corollary 2.11 cover the corresponding result given in [7] for the autonomous case. However, this does not rob the significance of [7] as it deals with general nonautonomous systems. From the proof of Theorem 2.9 given in the next section, it will be clear that Condition 2.1 for all $i \in J$ guarantees the existence of a common point $x^{*}$ of the $\gamma_{i}, i \in J$, such that $x_{i}^{*} \in\left(0,1 / a_{i i}\right]$ for $i \in J$ and $x_{i}^{*}=0$ otherwise. The purpose of (2.9) is to ensure that $x^{*}$ is not below $\gamma_{j}$ for any $j \in I_{N} \backslash J$ without actually finding $x^{*}$ and then calculating $\alpha_{j} x^{*}$ as Ahmad and Lazer [2] did for $|J|=N-1$.

The special case of Theorem 2.9 and Corollary 2.11 when $|J|=1$ can be stated as follows.

Corollary 2.14. Let $i \in I_{N}$ be fixed. Assume that

$$
a_{i i} \leq a_{k i} \quad\left(k \in I_{N} \backslash\{i\}\right)
$$

and either (2.8) for $j \in I_{N} \backslash\{i\}$ or Condition 2.1 is met. Then $Y^{\{i\}}$ is a global attractor of (1.1).

Remark 2.15. Condition (1.3) with $r=1$ can be written

$$
\forall k>1, \quad \exists i_{k}<k \quad \ni \quad \forall j \leq k, \quad a_{i_{k} j}<a_{k j} .
$$

Zeeman [9] and Montes de Oca and Zeeman [8] show the above conclusion with $i=1$ under the solo condition (2.11) without the requirement of (1.2) for $i=1$. As 
3638

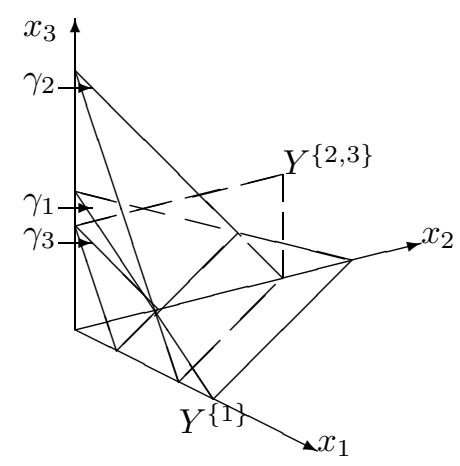

ZHANYUAN HOU

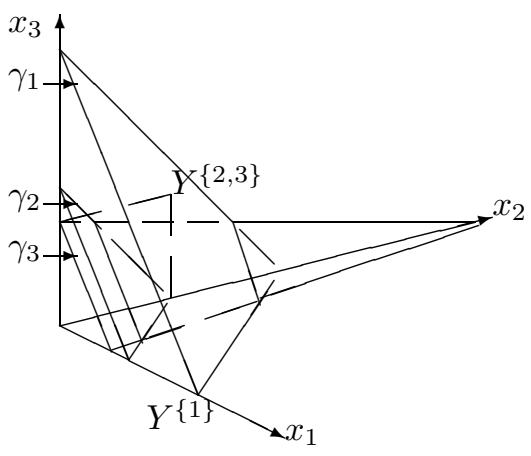

Figure 2. The left (right) satisfies (2.11) (the condition of Corollary 2.14 for $i=1$ ) but does not satisfy Condition 2.1 for $i=1$ $((2.11)$ for $k=3)$.

Corollary 2.14 requires either (2.8) or Condition 2.1, it can be seen that neither of (2.11) and the condition of Corollary 2.14 implies the other (see Figure 2). Again, we point out that the result in [8] is more general as it is mainly for nonautonomous systems.

\section{The proofs of Theorems 2.6 And 2.9}

Lemma 3.1. If (2.8) holds for all $i, j \in I_{N}$ with $i \neq j$, then $\bigcap_{i=1}^{N} \gamma_{i}=\left\{x^{*}\right\}$ with $0<x_{i}^{*} \leq a_{i i}^{-1}$ for $i \in I_{N}$.

Proof. Denoting the statement of this lemma by $P(N)$, we show the truth of $P(N)$ by induction. If (2.8) holds for all $i, j \in I_{N}$ with $i \neq j$, then

$$
\alpha_{i} Y^{I_{N-1} \backslash\{i\}}<1 \quad\left(i \in I_{N-1}\right) .
$$

Viewing $\pi_{N}$ as $\mathbb{R}_{+}^{N-1}$ and (3.1) as (1.2), where

$$
\pi_{k}=\left\{x \in \mathbb{R}_{+}^{N}: x_{k}=0\right\} \quad\left(k \in I_{N}\right),
$$

by Remark 2.5 we obtain

$$
\max \left\{0, \frac{a_{i j}}{a_{j j}}\left(1-\alpha_{j} Y^{I_{N-1} \backslash\{i, j\}}\right)\right\}<1-\alpha_{i} Y^{I_{N-1} \backslash\{i, j\}}
$$

for all $i, j \in I_{N-1}$ with $i \neq j$. Suppose $P(N-1)$ is true. Then $\left.\bigcap_{i=1}^{N-1} \gamma_{i}\right|_{x_{N}=0}=\left\{z^{*}\right\}$, i.e., $\left(\bigcap_{i=1}^{N-1} \gamma_{i}\right) \bigcap \pi_{N}=\left\{\left(z^{* T}, 0\right)^{T}\right\}$ where $z_{i}^{*} \in\left(0, a_{i i}^{-1}\right]$ for $i \in I_{N-1}$. Let $A_{0}=$ $\left(a_{i j}\right)_{(N-1) \times(N-1)}$ with $i, j \in I_{N-1}$. It follows from the uniqueness of $z^{*}$ that $A_{0}^{-1}$ exists. For $y_{N} \in\left[0, a_{N N}^{-1}\right]$, denoting the solution of the system $\alpha_{i} y=1\left(i \in I_{N-1}\right)$ by $y=\left(z\left(y_{N}\right)^{T}, y_{N}\right)^{T}$, we have

$$
z\left(y_{N}\right)=z^{*}-y_{N} A_{0}^{-1}\left(a_{i N}\right)_{(N-1) \times 1} .
$$

Since $\left(z(0)^{T}, 0\right)^{T}=\left(z^{* T}, 0\right)^{T} \in\left[Y^{\emptyset}, Y^{I_{N-1}}\right] \cap\left(\bigcap_{k \in I_{N-1}} \gamma_{k}\right)$, by Lemma 2.4 (iii) and Remark 2.3 for $i=N, \alpha_{N}\left(z(0)^{T}, 0\right)^{T}<1$. Let $x^{*}=\left(z\left(y_{N}^{*}\right)^{T}, y_{N}^{*}\right)^{T}$, where

$$
y_{N}^{*}=\sup \left\{y_{N} \in\left(0, a_{N N}^{-1}\right]: z\left(y_{N}\right) \in \operatorname{int} \mathbb{R}_{+}^{N-1}, \alpha_{N}\left(z\left(y_{N}\right)^{T}, y_{N}\right)^{T}<1\right\} \text {. }
$$


Then $x^{*} \in \bigcap_{i=1}^{N-1} \gamma_{i}$ with $x_{N}^{*}=y_{N}^{*} \in\left(0, a_{N N}^{-1}\right]$. Thus, as $a_{i i} z_{i}\left(y_{N}^{*}\right) \leq \alpha_{i} x^{*}=1$ for $i \in I_{N-1}, x^{*} \leq Y$. Then $P(N)$ is true if we can show that

$$
z\left(y_{N}^{*}\right) \in \operatorname{int} \mathbb{R}_{+}^{N-1} \text { and } \quad \alpha_{N} x^{*}=1 .
$$

Note that $z\left(a_{N N}^{-1}\right) \notin \mathbb{R}_{+}^{N-1}$ if $\alpha_{N}\left(z\left(a_{N N}^{-1}\right)^{T}, a_{N N}^{-1}\right)<1$. Hence, if (3.4) does not hold, then we must have $z\left(y_{N}^{*}\right) \in \partial \mathbb{R}_{+}^{N-1}$ so that $z_{j}\left(y_{N}^{*}\right)=0$ for some $j \in I_{N-1}$. Thus $x^{*} \in\left[Y^{\emptyset}, Y^{I_{N} \backslash\{j\}}\right] \cap \gamma_{j}$ for this $j \neq N$. By Remark 2.3, $x^{*}$ is above $\gamma_{N}$. This is impossible as (3.3) indicates that $x^{*}$ is on or below $\gamma_{N}$. We therefore have shown the truth of $P(N)$ when $P(N-1)$ is true.

For $N=2$, (2.8) for $i \in I_{N}$ becomes $a_{12} a_{22}^{-1}<1$ and $a_{21} a_{11}^{-1}<1 . P(2)$ is true since the system of $a_{11} x_{1}+a_{12} x_{2}=1$ and $a_{21} x_{1}+a_{22} x_{2}=1$ has a unique solution $x^{*} \in \mathbb{R}_{+}^{2}$ with $x_{i}^{*} \in\left(0, a_{i i}^{-1}\right]\left(i \in I_{2}\right)$. By induction, $P(N)$ is true for all $N \geq 2$.

In the following, by saying that $x$ is a solution of (1.1) we mean $x(0) \in \operatorname{int} \mathbb{R}_{+}^{N}$ and $x(t)$ satisfies (1.1) for all $t \geq 0$.

Lemma 3.2. Let $\hat{x} \in \mathbb{R}_{+}^{N}$ such that $\hat{x}_{i}=0$ if $\alpha_{i} \hat{x}>1, i \in I_{N}$, and let $\hat{y} \in \mathbb{R}_{+}^{N}$ be given by

$$
\hat{y}_{j}=\max \left\{0, \hat{x}_{j}+\left(1-\alpha_{j} \hat{x}\right) \frac{1}{a_{j j}}\right\} \quad\left(j \in I_{N}\right) .
$$

If a solution of (1.1) satisfies $\underline{\lim }_{t \rightarrow \infty} x(t) \geq \hat{x}$, then $\overline{\lim }_{t \rightarrow \infty} x(t) \leq \hat{y}$.

Proof. We show the conclusion by starting with

$$
\exists i \in I_{N}, \quad \varlimsup_{t \rightarrow \infty} x_{i}(t)=p>\hat{y}_{i}
$$

and then ending up with a contradiction. Take $\delta=\frac{1}{4} a_{i i}\left(p-\hat{y}_{i}\right)$ and $\varepsilon>0$ such that $\varepsilon \alpha_{i} \hat{x}<\delta$. Then, by the assumption, there is a $T \geq 0$ such that $x(t) \geq(1-\varepsilon) \hat{x}$ for $t \geq T$. Hence, if $x_{i}(t) \geq \frac{1}{4}\left(3 p+\hat{y}_{i}\right)$ for some $t \geq T$, from (3.5) we obtain

$$
\alpha_{i} x(t) \geq a_{i i} \hat{y}_{i}+3 \delta+(1-\varepsilon) \alpha_{i} \hat{x}^{I_{N} \backslash\{i\}} \geq 1-\varepsilon \alpha_{i} \hat{x}^{I_{N} \backslash\{i\}}+3 \delta>1+2 \delta
$$

so that

$$
\dot{x}_{i}(t)<-2 \delta b_{i} x_{i}(t)
$$

This, along with (3.6), leads to $x_{i}(t) \geq p>\frac{1}{4}\left(3 p+\hat{y}_{i}\right)$ and further to (3.7) for all $t \geq T$. Integration of (3.7) gives $\lim _{t \rightarrow \infty} x_{i}(t)=0$, a contradiction to (3.6). Therefore, $\varlimsup_{t \rightarrow \infty} x(t) \leq \hat{y}$.

Lemma 3.3. Assume that

(i) $\hat{x}, \hat{y} \in \mathbb{R}_{+}^{N}$ as in Lemma 3.2;

(ii) every solution of (1.1) satisfies $\varliminf_{t \rightarrow \infty} x(t) \geq \hat{x}$;

(iii) for some $i \in I_{N}, \alpha_{i} \hat{x}<1$ and $\gamma_{i} \cap[\hat{x}, \hat{z}]$, if not empty, is above every $\gamma_{j}(j \neq i)$, where $\hat{z}=\hat{y}^{I_{N} \backslash\{i\}}+\hat{x}^{\{i\}}$.

Then there is a $\delta>0$ such that every solution of (1.1) satisfies

$$
{\underline{\lim _{t \rightarrow \infty}}}_{t}(t) \geq \hat{x}_{i}+\delta .
$$


Proof. Put

$$
\begin{gathered}
\varepsilon=\sup \left\{\alpha_{i} x: x \in\left(\bigcup_{j \in I_{N} \backslash\{i\}} \gamma_{j} \cup\{\hat{x}\}\right) \cap[\hat{x}, \hat{z}]\right\}, \\
\Gamma_{i}=\left\{x \in \mathbb{R}_{+}^{N}: \alpha_{i}\left(x^{I_{N} \backslash\{i\}}+\hat{x}^{\{i\}}\right) \geq \frac{1}{2}(1+\varepsilon)\right\} .
\end{gathered}
$$

Then, by (iii) and the compactness of the set in (3.9), $\varepsilon \in[0,1)$. We show that $\delta=\frac{1}{2}(1-\varepsilon) a_{i i}^{-1}$ meets the requirement of (3.8). For any solution $x$ of (1.1), we shall see later the existence of $T \geq 0$ such that

$$
\alpha_{i}\left(x(t)^{I_{N} \backslash\{i\}}+\hat{x}^{\{i\}}\right)<\frac{1}{2}(1+\varepsilon)
$$

for all $t \geq T$. Then, for any $t \geq T, x(t)$ is below $\gamma_{i}$ (i.e., $\alpha_{i} x(t)<1$ ) if $x_{i}(t) \leq$ $\hat{x}_{i}+\delta$. If (3.8) does not hold, since $\dot{x}_{i}(t)>0$ if and only if $x(t)$ is below $\gamma_{i}$, we must have $x_{i}(t) \leq \delta_{0}$ for some $\delta_{0}<\hat{x}_{i}+\delta$ and all $t \geq T$. By $(3.11), \alpha_{i} x(t)<$ $\frac{1}{2}(1+\varepsilon)+a_{i i}\left(x_{i}(t)-\hat{x}_{i}\right) \leq \frac{1}{2}(1+\varepsilon)+a_{i i}\left(\delta_{0}-\hat{x}_{i}\right) \equiv \varepsilon_{0}<1$. Integration of the $i$ th component equation of (1.1) leads to $\lim _{t \rightarrow \infty} x_{i}(t)=\infty$, which contradicts $x_{i}(t) \leq \delta_{0}$. Therefore, we have shown (3.8).

The existence of $T \geq 0$ such that (3.11) holds for $t \geq T$ follows from (i), (ii) and Lemma 3.2 if $\alpha_{i} \hat{z}<\frac{1}{2}(1+\varepsilon)$. Suppose $\alpha_{i} \hat{z} \geq \frac{1}{2}(1+\varepsilon)$. Then $\hat{z} \in \Gamma_{i}$ and $[\hat{x}, \hat{y}] \cap \Gamma_{i} \supset[\hat{x}, \hat{z}] \cap \Gamma_{i} \neq \emptyset$. The definitions (3.9) and (3.10) suggest that $[\hat{x}, \hat{y}] \cap \Gamma_{i}$ is above every $\gamma_{j}(j \neq i)$, so

$$
\eta \equiv \inf \left\{\alpha_{j} y: y \in[\hat{x}, \hat{y}] \cap \Gamma_{i}, j \in I_{N} \backslash\{i\}\right\}>1 .
$$

Then (i), (ii) and Lemma 3.2 lead to the existence of $T_{0} \geq 0$ such that, for any $t \geq T_{0}$ and $j \in I_{N} \backslash\{i\}, \alpha_{j} x(t) \geq \frac{1}{2}(1+\eta)>1$ so that $\dot{x}_{j}(t) \leq-\frac{1}{2} b_{j}(\eta-1) x_{j}(t)$ as long as $x(t) \in \Gamma_{i}$. This indicates that either there is a $T \geq T_{0}$ such that $x(t) \notin \Gamma_{i}$ for $t \geq T$ or $x(t) \in \Gamma_{i}$ for all $t \geq T_{0}$. In the latter case, $\lim _{t \rightarrow \infty} x(t)^{I_{N} \backslash\{i\}}=0$ so that $\alpha_{i} \hat{x}^{\{i\}} \geq \frac{1}{2}(1+\varepsilon)$. This is impossible as, by (3.9), $\alpha_{i} \hat{x}^{\{i\}} \leq \alpha_{i} \hat{x} \leq \varepsilon<\frac{1}{2}(1+\varepsilon)$. Hence, by the equivalence of $x(t) \notin \Gamma_{i}$ to (3.11), $x(t)$ satisfies (3.11) for $t \geq T$.

Proof of Theorem 2.9. Since Condition 2.1 holds for all $i \in J$, by Remark 2.3 $\left[Y^{\emptyset}, Y^{I_{N} \backslash\{i\}}\right] \cap \gamma_{i}$, if not empty, is above every $\gamma_{j}(j \neq i)$. Then, as $\left[Y^{\emptyset}, Y^{J \backslash\{i\}}\right] \subset$ $\left[Y^{\emptyset}, Y^{I_{N} \backslash\{i\}}\right],\left[Y^{\emptyset}, Y^{J \backslash\{i\}}\right] \cap \gamma_{i}$ is above every $\gamma_{j}(j \neq i)$ if $\left[Y^{\emptyset}, Y^{J \backslash\{i\}}\right] \cap \gamma_{i} \neq \emptyset$. Note that $\left[Y^{\emptyset}, Y^{J}\right] \subset \bigcap_{k \in I_{N} \backslash J} \pi_{k}$. Viewing $\bigcap_{k \in I_{N} \backslash J} \pi_{k}$ as $\mathbb{R}_{+}^{|J|}$ and applying Lemmas 2.4 and 3.1 to the $\gamma_{i}(i \in J)$ when $|J|>1$, we have

$$
\left(\bigcap_{i \in J} \gamma_{i}\right) \cap\left(\bigcap_{k \in I_{N} \backslash J} \pi_{k}\right)=\left\{x^{*}\right\},
$$

where $x_{i}^{*} \in\left(0, a_{i i}^{-1}\right]$ for $i \in J$ and $x_{i}^{*}=0$ otherwise. That this also holds for $|J|=1$ is trivial.

By Lemma 3.2, every solution of (1.1) satisfies

$$
Y^{\emptyset} \leq \underline{\lim }_{t \rightarrow \infty} x(t) \leq \varlimsup_{t \rightarrow \infty} x(t) \leq Y .
$$

Applying Lemma 3.3 to all $i \in J$, we have a $\delta \in(0,1)$ such that every solution of (1.1) satisfies $\underline{\lim }_{t \rightarrow \infty} x(t) \geq \delta x^{*}$. Let $\hat{x}=\delta x^{*}$ and let $\hat{y}$ be given by (3.5). Then, by Lemma 3.2 again, every solution of (1.1) satisfies

$$
\hat{x} \leq \underline{\lim }_{t \rightarrow \infty} x(t) \leq \varlimsup_{t \rightarrow \infty} x(t) \leq \hat{y} .
$$


If we can show that $\hat{x}=x^{*}$, then, since $\alpha_{i} x^{*}=1$ for $i \in J$ and $\alpha_{i} x^{*} \geq 1$ for $i \in I_{N} \backslash J$ by (2.9), (3.5) gives $\hat{y}=x^{*}$ so that $x^{*}$ is a global attractor. For this purpose, we put

$$
\delta_{0}=\sup \{\delta \in(0,1):(3.12) \text { holds for all solutions of }(1.1)\}
$$

and show that $\delta_{0}=1$ for (3.12) still holds with $\hat{x}=\delta_{0} x^{*}$.

Suppose $\delta_{0}<1$. Then, for each $i \in J$, since $\alpha_{i} \hat{x}=\delta_{0} \alpha_{i} x^{*}=\delta_{0}<1$, if we can show that

$$
\left[\hat{x}, \hat{x}^{\{i\}}+\hat{y}^{I_{N} \backslash\{i\}}\right] \cap \gamma_{i} \quad \text { is above every } \gamma_{j}(j \neq i)
$$

when $\left[\hat{x}, \hat{x}^{\{i\}}+\hat{y}^{I_{N} \backslash\{i\}}\right] \cap \gamma_{i} \neq \emptyset$, by Lemma 3.3 we can find a $\delta \in\left(\delta_{0}, 1\right)$ such that (3.12) holds with $\hat{x}=\delta x^{*}$. This contradicts (3.13). Thus $\delta_{0}=1$.

To verify (3.14) under the hypothesis $\delta_{0}<1$, we introduce a mapping $f: \mathbb{R}_{+}^{N} \rightarrow$ $\mathbb{R}_{+}^{N}$ given by $f(x)=\hat{x}+\left(1-\delta_{0}\right) x$. Then $f\left(\left[Y^{\emptyset}, Y^{I_{N} \backslash\{i\}}\right]\right)=\left[\hat{x}, f\left(Y^{I_{N} \backslash\{i\}}\right)\right]$. Since the definition of $x^{*}$ and (2.9) imply that $\alpha_{j} x^{*} \geq 1$ for all $j \in I_{N}$, we have $f_{i}\left(Y^{I_{N} \backslash\{i\}}\right)=\hat{x}_{i}$ and, by $(3.5)$,

$$
f_{j}\left(Y^{I_{N} \backslash\{i\}}\right)=\hat{x}_{j}+\left(1-\delta_{0}\right) \frac{1}{a_{j j}} \geq \max \left\{0, \hat{x}_{j}+\left(1-\delta_{0} \alpha_{j} x^{*}\right) \frac{1}{a_{j j}}\right\}=\hat{y}_{j}
$$

for $j \in I_{N} \backslash\{i\}$. Thus $\left[\hat{x}, \hat{x}^{\{i\}}+\hat{y}^{I_{N} \backslash\{i\}}\right] \subseteq f\left(\left[Y^{\emptyset}, Y^{I_{N} \backslash\{i\}}\right]\right)$. Therefore, instead of showing (3.14), we need only prove that

$$
f\left(\left[Y^{\emptyset}, Y^{I_{N} \backslash\{i\}}\right]\right) \cap \gamma_{i} \text { is above every } \gamma_{j}(j \neq i)
$$

if $f\left(\left[Y^{\emptyset}, Y^{I_{N} \backslash\{i\}}\right]\right) \cap \gamma_{i} \neq \emptyset$. For any $y \in f\left(\left[Y^{\emptyset}, Y^{I_{N} \backslash\{i\}}\right]\right) \cap \gamma_{i}$, there is a unique $x \in\left[Y^{\emptyset}, Y^{I_{N} \backslash\{i\}}\right]$ such that $f(x)=y$. As $\delta_{0}<1$ and

$$
1=\alpha_{i} y=\alpha_{i} f(x)=\alpha_{i}\left\{\delta_{0} x^{*}+\left(1-\delta_{0}\right) x\right\}=\delta_{0}+\left(1-\delta_{0}\right) \alpha_{i} x,
$$

we have $\alpha_{i} x=1$ so that $x \in\left[Y^{\emptyset}, Y^{I_{N} \backslash\{i\}}\right] \cap \gamma_{i}$. By Condition 2.1 and Remark 2.3, $\alpha_{j} x>1$ and this, along with $\alpha_{j} x^{*} \geq 1$, implies that

$$
\alpha_{j} y=\alpha_{j} f(x)=\delta_{0} \alpha_{j} x^{*}+\left(1-\delta_{0}\right) \alpha_{j} x>\delta_{0}+\left(1-\delta_{0}\right)=1 .
$$

Thus (3.15) holds if $f\left(\left[Y^{\emptyset}, Y^{I_{N} \backslash\{i\}}\right]\right) \cap \gamma_{i} \neq \emptyset$.

Proof of Theorem 2.6. The proof of Theorem 2.9 is still valid with $J=I_{N}$.

\section{REFERENCES}

1. S. Ahmad. On the Nonautonomous Voterra-Lotka Competition Equations, Proc. Amer. Math. Soc. 117 (1993), 199-205. MR 93c:34109

2. S. Ahmad and A. C. Lazer. One Species Extinction in an Autonomous Competition Model, Proc. Fisrt World Congress on Nonlinear Analysis, Walter DeGruyter, Berlin, 1995. CMP 96:12

3. S. Ahmad and A. C. Lazer. On the Nonautonomous N-Competing Species Problem, Appl. Anal. 57 (1995), 209-223. MR 97a:34128

4. K. Gopalsamy. Stability and Oscillations in Delay Differential Equations of Population Dynamics, Kluwer Academic Publishers, Dordrecht, 1992. MR 93c:34150

5. K. Gopalsamy and R. A. Ahlip. Time Delays in N-Species Competition-I: Global Stability in Constant environments, Bull. Austral. Math. Soc. 27 (1983), 427-441. MR 84k:45016

6. M. Kaykobad. Positive Solutions of Positive Linear Systems, Lin. Alg. Appl. 64 (1985), 133140. MR 86e: 92026

7. F. Montes de Oca and M. L. Zeeman. Balancing Survival and Extinction in Nonautonomous Competitive Lotka-Volterra Systems, J. Math. Anal. Appl. 192 (1995), 360-370. MR 96c:92017 
8. F. Montes de Oca and M. L. Zeeman. Extinction in Nonautonomous Competitive LotkaVolterra Systems, Proc. Amer. Math. Soc. 124 (1996), 3677-3687. MR 97b:92017

9. M. L. Zeeman. Extinction in Competitive Lotka-Volterra Systems, Proc. Amer. Math. Soc. 123 (1995), 87-96. MR 95c:92019

10. P. van den Driessche and M. L. Zeeman. Three-Dimensional Competitive Lotka-Volterra Systems with no Periodic Obits, SIAM J. Appl. Math. 58 (1998), 227-234. CMP 98:10

Department of Computing, Information Systems and Mathematics, London Guildhall University, 100 Minories, London EC3N 1JY, United Kingdom

E-mail address: hou@lgu.ac.uk 